\title{
Ventricular Electrical Standstill in a Patient with Left Ventricular Assist Device
}

\section{Chee Kiang Teoh ${ }^{1 *}$, Kah Leng Ho ${ }^{2}$, Louis Teo², David Sim², Ka Lee Kerk ${ }^{3}$, Jia Lin Soon ${ }^{4}$, Teing Ee Tan ${ }^{4}$, Sivathasan Cumaraswamy ${ }^{4}$ and Choon Pin Lim²}

${ }^{1}$ Department of Cardiology, National Heart Institute, Malaysia

${ }^{2}$ Department of Cardiology, National Heart Centre Singapore, Singapore

${ }^{3}$ Mechanical Circulatory Support, Heart and Lung Transplant Unit, National Heart Centre Singapore, Singapore

${ }^{4}$ Department of Cardiothoracic Surgery, National Heart Centre Singapore, Singapore

*Corresponding author: Chee Kiang Teoh, $\mathrm{MRCP}(U K)$, Department of Cardiology, National Heart Institute, 145, Jalan Tun Razak, 50400 Kuala Lumpur, Malaysia, Tel: (+60)-326178200, Fax: (+60)-326928395, E-mail: ckteoh.hsp@gmail.com

\begin{abstract}
Left ventricular assist device (LVAD) is designed primarily for circulatory support in left ventricular failure and its function relies heavily on intrinsic right ventricular function. We report, to our knowledge, the longest reported case of ventricular electrical standstill from progressive cardiomyopathy who was successfully managed on LVAD. A 51-yearold man, non-ischaemic cardiomyopathy who had been on HeartMate II LVAD support for three years, presented with frequent intermittent low pump flow together with mild reduced effort tolerance. His electrocardiogram showed ventricular standstill. Echocardiogram confirmed absent of left and right ventricular contraction. No spontaneous nor inducible electrical activity was seen with catheter manipulation during electrophysiology study. Careful stepwise increment of LVAD pump speed generated adequate physiological output in his Fontan mechanism of circulation, successfully bridging him to heart transplant.
\end{abstract}

\section{Keywords}

Ventricular electrical standstill, Cardiomyopathy, Right ventricular failure, Left ventricular assist device, Low pump flow

\section{Abbreviations}

VES: Ventricular Electrical Standstill; CM: Cardiomyopathy; LVAD: Left Ventricular Assist Device; LPF: Low Pump Flow; PA: Prolonged Asystole; RVF: Right Ventricular Failure

\section{Introduction}

Left ventricular assist device (LVAD) is designed primarily for circulatory support in left ventricular failure.
LVAD function relies heavily on right ventricular function because LVAD filling pressure and pump output will be compromised without adequate preload returning from the pulmonary bed. As a result, right heart failure can lead to various problems including systemic hypoperfusion, multi-organt failure and recurrent heart failure hospitalization. Most LVAD patients undergo heart transplantation after short to intermediate term LVAD support, while patients without it often demise from LVAD-related complications such as stroke and infection. The long term consequences of progressive, on-going cardiomyopathy in LVAD patient remains unknown. We report a case of a patient on long-term LVAD support developing ventricular electrical standstill from progressive cardiomyopathy.

\section{Case Description}

A 51-year-old man, with complete heart block and non-ischaemic cardiomyopathy who had undergone prior Atrial Septal Defect device closure and Cardiac Resynchronization Therapy-Defibrillator implantation, underwent HeartMate II LVAD implantation as a bridge to heart transplant therapy due to refractory heart failure. After 23 months of LVAD support, he developed new, recurrent heart failure symptoms, with echocardiographic findings of severe aortic insufficiency. He then underwent surgical closure of aortic valve with a simple central coaptation stitch [1]. Thereafter, he was clinical- 


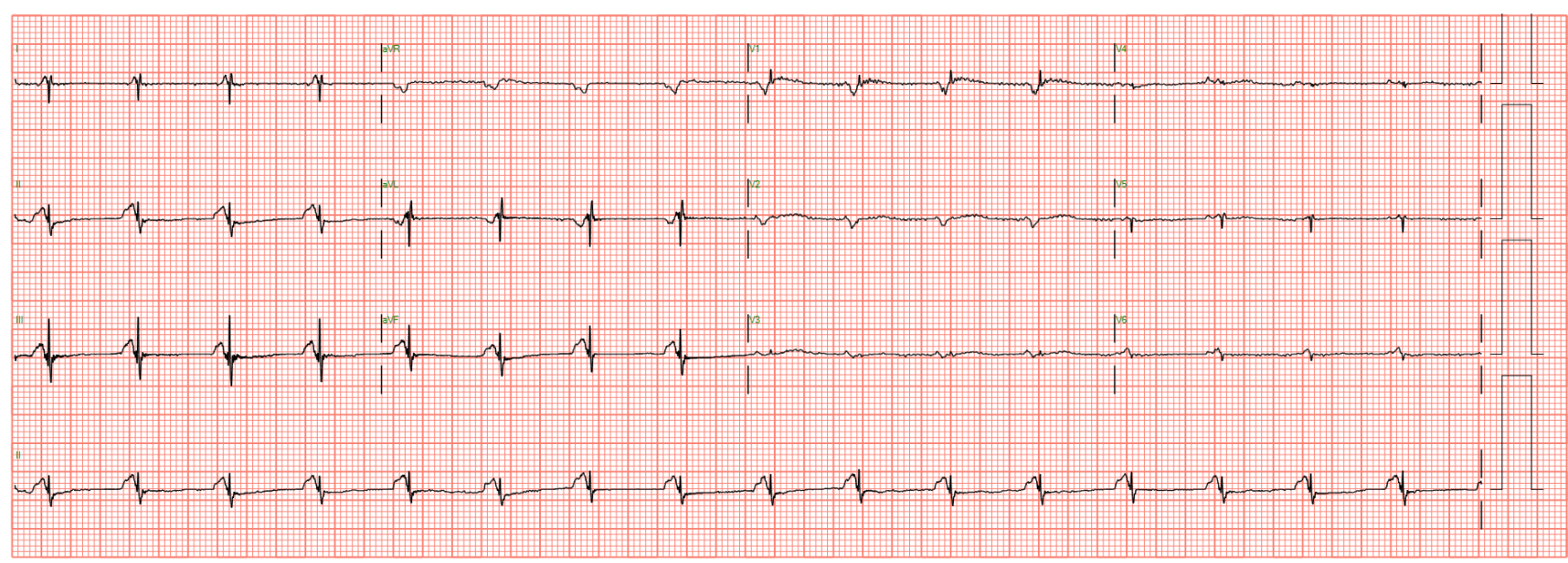

Figure 1: Electrocardiogram shows ventricular standstill.

a

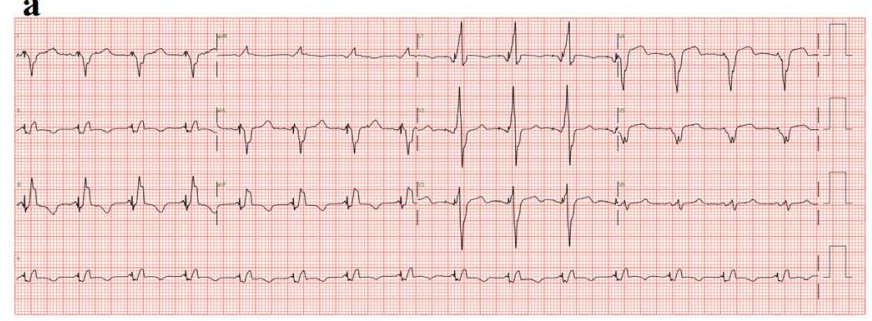

b

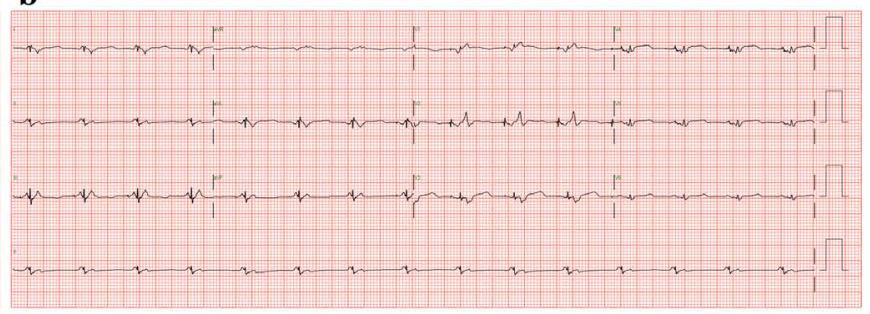

Figure 2: Sequential electrocardiograms over the course of 1 year.

ly well with no residual aortic regurgitation. However, at 38 months of LVAD support, he presented with frequent LVAD alarms due to intermittent low pump flow for the past 3 weeks. He reported slight reduction in effort tolerance with New York Heart Association (NYHA) functional class II. Apart from raised jugular venous pressure and mild bilateral pedal edema, there was no other overt signs of right ventricular failure such as ascites, pulsatile hepatomegaly or abnormal liver function test. His baseline serum creatinine had deteriorated to $141 \mathrm{umol} / \mathrm{L}$ from a baseline of $97 \mathrm{umol} / \mathrm{L}$. His baseline lactate dehydrogenase (LDH) was $608 \mathrm{U} / \mathrm{L}$. Electrocardiogram (ECG) showed intrinsic atrial rhythm with ventricular pacing spikes without evidence of ventricular capture despite maximal output, consistent with ventricular electrical standstill. There was no intrinsic escape R wave (Figure 1). Echocardiography demonstrated absent left and right ventricular contraction with preserved atrial contraction. The interventricular septum was in midline position. Both ventricles were dilated-left ventricle measured $6.1 \mathrm{~cm}$ while right ventricle inlet measured 5.5 $\mathrm{cm}$. Invasive hemodynamic assessment revealed a cardiac index of $2.1 \mathrm{~L} / \mathrm{min} / \mathrm{m}^{2}$, central venous pressure of $20 \mathrm{mmHg}$, mean pulmonary arterial pressure $22 \mathrm{mmHg}$, wedge pressure $15 \mathrm{mmHg}$ and pulmonary vascular resistance of 1.6 wood units. Subsequent Electrophysiology study (EPS) involving right ventricular mapping was performed with 3D electroanatomical mapping using point by point mapping with scar defined with endocardial bipolar voltage set at $0.5 \mathrm{mv}-1.5 \mathrm{mV}$. This exhibited extensive scar over his right ventricle. No spontaneous nor inducible electrical activity was seen with catheter manipulation. LVAD pump speed was increased stepwise to $10,000 \mathrm{rpm}$ from a baseline of 9,200 rpm with echocardiographic guidance. He required only a small dose of frusemide $20 \mathrm{mg}$ daily. He was closely followed up with regular outpatient reviews and phone call monitoring so that more aggressive treatment can be instituted at the earliest sign of systemic congestion. Fortunately, the patient reported an improvement in clinical symptoms with no further low flow LVAD alarms. Serum creatinine recovered back to $97 \mathrm{umol} / \mathrm{L}$. With the increase in LVAD pump speed, his LDH increase to a peak of 894 $\mathrm{U} / \mathrm{L}$ before coming down to $570 \mathrm{U} / \mathrm{L}$ on repeat testing six weeks later. He was in NYHA functional class I to II and returned back to normal daily activities, including full-time employment, for 111 days without any further alarms of low flow state prior to undergoing orthotopic heart transplantation. Unfortunately, he succumbed to septicemic shock secondary to serratia and pseudomonas infection 7 days after the transplant.

\section{Discussion}

This case illustrated two important learning points. Firstly, there was a progression of the patient's cardiomyopathy in spite of left ventricular decompression by the LVAD, leading to ongoing myocardial fibrosis and eventually an electrically silent heart. This was evidenced by the ventricular standstill on his ECG without intrinsic escape ventricular beats as well as scar demonstrated by low voltage on EPS. Review of previous ECGs showed a progressive diminution in the amplitude of 
QRS complexes over the last 1 year suggesting active progressive myopathic process (Figure 2a and Figure 2b). CRT-D check when he presented showed no capture in the right and left ventricular leads. Before this, lead impedance and pacing threshold checks of the right atrial, right ventricular and left ventricular leads have always been within normal limits. Cardiogenetic testing was also unremarkable. In retrospect, core biopsy during LVAD implantation only showed focal transmural fibrosis with no evidence of Giant Cells, inflammatory or infiltrative disease. The patient was maintained on guideline-directed heart failure therapy while on LVAD support which did not arrest the decline. Nonetheless, LVAD was still capable of maintaining his circulatory support.

The EP study was performed to identify whether it was a global myocardial problem or a result of a CRT exit site block. Additionally it would also help identify a favourable position to resite the right ventricular lead. In instances with localized exit block, there should be demonstrable intrinsic slow ventricular escape complexes from remaining viable electrical system seen on the ECG. The absence of intrinsic ventricular complexes suggested a potential global problem. The lack of inducible electrical activity during catheter manipulation suggested the presence of transmural infarct. The scar burden of the left ventricle was not evaluated as we had expected that this is likely to have been just as extensive and would not have affected further clinical management.

Histology of the patient's explanted heart at transplantation provided intriguing insights. As expected, there was extensive transmural fibrosis. Interestingly, extensive and advanced atherosclerosis involving all major coronary arterial branches was found. Coronary angiogram was not pursued in the workup because he was noted to have only a $30 \%$ stenosis in the mid left anterior descending artery while the left circumflex and right coronary arteries were completely normal just prior to LVAD implantation. While progression of the underlying non-ischemic cardiomyopathy could have resulted in fibrosis of the entire myocardium over time, the development of extensive atherosclerosis in a previously relatively disease-free coronary anatomy can certainly contribute to the decline. This occurred even while the patient remained on LVAD support. Moreover, this extent of coronary atherosclerosis was not seen previously in other patients with dilated cardiomyopathy undergoing heart transplantation in our center. A potential hypothesis that could be generated from this case is that surgical closure of the aortic valve might have contributed to the accelerated atherosclerosis in this patient. This case also highlights the importance of considering coronary atherosclerosis as a cause of worsening right or left heart function even in LVAD patients with previously documented absence of significant coronary artery disease.
Secondly, in spite of a non-functioning right ventricle, our patient only presented with mild right ventricular failure symptoms. This was overcome by increasing the LVAD pump speed with frequent close monitoring. Pump flow when he first presented with biventricular standstill was $3.1 \mathrm{~L} / \mathrm{min}$ at a pump speed of $9200 \mathrm{rpm}$. With stepwise increment of pump speed, pump flow increased to $3.6 \mathrm{~L} / \mathrm{min}$ at $10000 \mathrm{rpm}$. While the estimated pump flow increment was only $0.5 \mathrm{~L} / \mathrm{min}$ after an increase of pump speed of $800 \mathrm{rpm}$, we postulate that the increased pump speed helped reduce the wedge pressures and sufficiently improved peripheral perfusion leading to clinical improvement. To our knowledge, our patient is the longest reported case of a patient surviving on LVAD with biventricular standstill, longer than the two previously reported cases of LVAD patients with asystole for 15 days and 104 days respectively [2,3]. In our patient, even though atrial contraction was preserved, this probably provided negligible contribution to the circulation. In a typical LVAD patient with right ventricular failure, the left ventricular preload is usually poor due to restrictive flow return from pulmonary vascular bed. With prolonged LVAD support, the pulmonary circulation undergoes remodeling, often leading to a normalization of pulmonary vascular resistance [4]. The right ventricle and the pulmonary circulation now serve as a passive conduit, alike a Fontan circuit. Our patient was also maintained on Sildenafil since LVAD implantation, which would have aided in minimizing pulmonary vascular resistance [5] and therefore presented a favourable physiology when there was deterioration in biventricular function.

It is rare to see patients progress to such late stage of dilated cardiomyopathy even if they are on long term LVAD support. In high volume transplant centres, such patients would have received their heart transplants. Many patients on long term LVAD support may also succumb to complications of LVAD before reaching this stage. We postulate that as destination therapy LVAD becomes more common, such cases are likely to become more common and hope that the sharing of our experience can add to the literature in managing similar presentations.

\section{Conclusion}

Our patient demonstrated the progression of cardiomyopathy leading to electrical ventricular standstill. The diminution of ECG complexes in LVAD patients over time may indicate a progression of cardiomyopathy or coronary artery disease and warrant further investigation. It was still possible to maintain a stable Fontan mechanism of circulation in the setting of favourable pulmonary vascular resistance in such a patient by conventional LVAD for a considerable period of time with careful stepwise increment of LVAD pump speed.

\section{Financial Support}

This case report received no specific grant from any funding agency, commercial or not-for-profit sectors. 


\section{Acknowledgments}

None.

\section{Disclosures}

None.

\section{Disclaimer}

None.

\section{References}

1. McKellar SH, Deo S, Daly RC, Durham LA, Joyce LD, et al. (2014) Durability of central aortic valve closure in patients with continuous flow left ventricular assist devices. J Thorac Cardiovasc Surg 147: 344-348.

2. Takayama H, Aldea GS, Verrier ED, Salerno CT, Levy WC, et al. (2006) Successful management of asystole with an isolated left ventricular assist device. J Heart Lung Transplant 25: 490-492.

3. Javed W, Chaggar PS, Venkateswaran R, Shaw SM (2016) Prolonged asystole in a patient with an isolated left ventricular assist device. Future Cardiol 12: 533-538.

4. Atluri P, Fairman A, MacArthur JW, Goldstone AB, Cohen JE, et al. (2013) Continuous flow left ventricular assist device implant significantly improves pulmonary hypertension, right ventricular contractility and tricuspid valve competence. J Card Surg 28: 770-775.

5. Fang JC, DeMarco T, Givertz MM, Borlaug BA, Lewis GD, et al. (2012) World Health Organization Pulmonary Hypertension group 2: Pulmonary hypertension due to left heart disease in the adult-a summary statement from the Pulmonary Hypertension Council for the International Society for Heart And Lung Transplantation. J Heart Lung Transplant 31: 913-933. 